\title{
Delocalisation transition in chains with correlated disorder
}

\author{
Gerald Schubert $^{\mathrm{a}}$, Alexander Weiße ${ }^{\mathrm{b}}$, Holger Fehske ${ }^{\mathrm{a}, *}$ \\ a Institut für Physik, Ernst-Moritz-Arndt Universität Greifswald, 17487 Greifswald, Germany \\ ${ }^{\mathrm{b}}$ School of Physics, The University of New South Wales, Sydney NSW 2052, Australia
}

\begin{abstract}
We show that in the one-dimensional (1D) Anderson model long-range correlations within the sequence of on-site potentials may lead to a region of extended states in the vicinity of the band centre, i.e., to a correlation-induced insulator-metal transition. Thus, although still disordered, the 1D system can behave as a conductor.
\end{abstract}

Key words: disordered electron systems, Anderson metal-insulator transition

In the context of electronic transport in quasi-1D binary solids, polymer chains or biological molecules, recently systems with correlations in the sequence of on-site energies attracted increasing attention [1]. The examination of models with correlated disorder is also challenging from a theoretical point of view. In $1 \mathrm{D}$ noninteracting electron systems with independent random atomic potentials all states are exponentially localised at any amount of disorder [2]. Internal correlations within the potential landscape, however, might cause a breakdown of the Anderson localisation phenomenon in 1D and 2D [3,4]. Such long-range correlated random sequences without any intrinsic scale are observed in several stochastic processes in nature [5]. One of their characteristics is the power law decay of the Fourier transform of the two-point correlation function with the wavenumber of the random fluctuations, $\mathcal{F}\left(\left\langle\epsilon_{i} \epsilon_{j}\right\rangle\right) \sim 1 / k^{\alpha}$. Based on these findings de Moura and Lyra [4] proposed an Anderson type model, $H=\sum_{j} \epsilon_{j} c_{j}^{\dagger} c_{j}-t \sum_{\langle i j\rangle}\left[c_{i}^{\dagger} c_{j}+\right.$ H.c. $]$, with the following ansatz for the on-site potentials,

$$
\epsilon_{j}=\nu(\alpha) \sum_{k=1}^{N / 2} k^{-\alpha / 2} \cos \left(2 \pi j k / N+\phi_{k}\right),
$$

where the $N / 2$ random phases $\phi_{k}$ are uniformly distributed in the interval $[0,2 \pi]$ and $\alpha$ controls the strength of the correlation. The case $\alpha=0$ corresponds to strongest

\footnotetext{
* Corresponding Author: Phone: +49-3834-86-4760, Fax +49-3834-864701, Email: fehske@physik.uni-greifswald.de
}

disorder and is almost equivalent to the uncorrelated Anderson model with Gaussian distributed on-site potentials. In order to obtain comparable results each disordered sequence is normalised to variance $\sigma^{2}=1$, i.e. $\nu(\alpha)=$ $\left(\sum_{k=1}^{N / 2} k^{-\alpha} / 2\right)^{-1 / 2}$. This ensures that both disorder and bandwidth remain finite in the thermodynamic limit.

In the theoretical investigation of disordered systems it turned out that distribution functions for the random quantities of interest take the centre stage [6]. Of particular importance is the probability density $p\left(\rho_{i}(E)\right)$ of the local density of states (LDOS)

$$
\rho_{i}(E)=\sum_{n=1}^{N}\left|\psi_{n, i}\right|^{2} \delta\left(E-E_{n}\right) .
$$

For a given energy $E, \rho_{i}(E)$ measures the local amplitude of the wave function at Wannier site $i$ and therefore contains direct information about the localisation properties. Contrary to the mean (arithmetically averaged) density of states, $\rho_{\text {av }}(E)=\left\langle\rho_{i}(E)\right\rangle$, the LDOS becomes critical at the localisation transition. Concurrently $p\left(\rho_{i}(E)\right)$ was found to have essentially different properties for localised and extended phases [7]. Of course, the study of entire distributions is a bit inconvenient, and for practical calculations the so-called "typical DOS" (geometric average) $\rho_{\text {ty }}(E)=$ $\exp \left\langle\ln \rho_{i}(E)\right\rangle$ is frequently used. Since $\rho_{\text {ty }}(E)$ vanishes at the Anderson transition it acts as a kind of "order parameter".

Numerically the LDOS distribution can be easily calcu- 

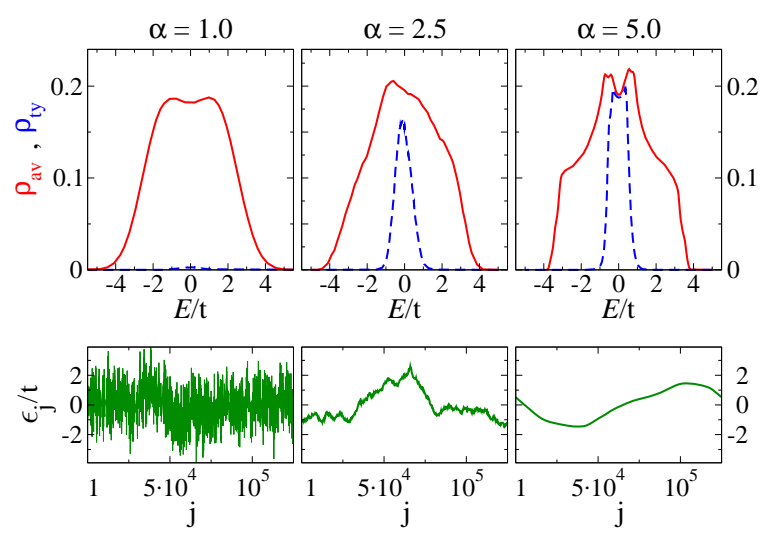

Fig. 1. Average (solid) and typical (dashed) DOS for the Anderson model with correlated disorder on a linear chain with 125000 sites and different correlation strengths $\alpha$. The typical DOS was calculated at $K_{s}=32$ sites for $K_{r}=32$ realizations of disorder using 32768 Chebyshev moments, whereas for the average DOS only 256 Chebyshev moments and a random initial vector were used. The lower panels show one characteristic sequence of atomic potentials $\epsilon_{j}$ for each value of $\alpha$.

lated with the kernel polynomial method [8]. The corresponding arithmetic and geometric averages $\rho_{\text {av }}$ and $\rho_{\text {ty }}$ are shown in Fig. 1. In contrast to the uncorrelated Anderson model we observe the following effects: (i) most notably, while for small values of $\alpha$ the typical DOS vanishes for the entire band, with stronger correlation a region of extended states appears near the band centre; (ii) for larger values of $\alpha$ a dip in $\rho_{\text {av }}$ develops at the band centre, which might be a signature of the 1D DOS of the completely ordered system that is expected for infinitely strong correlation; (iii) even though on average the model is symmetric with respect to $E \rightarrow-E$ in Fig. 1 the averaged density of states shows a noticeable asymmetry for intermediate values of $\alpha$. We fear that, due to the longer ranged fluctuations within the on-site potentials for increasing $\alpha$, the considered rather large ensemble $(32 \times 32$ realizations $)$ is still insufficient to achieve a proper statistics.

Figure 2 illustrates the behaviour of the probability distribution $P\left(\rho_{i}\right)=\int_{0}^{\rho_{i}} p\left(\rho_{i}^{\prime}\right) d \rho_{i}^{\prime}$. At small values of $\alpha, P\left(\rho_{i}\right)$ reflects the characteristics of the LDOS distribution of localised states, i.e. large weight on small values of $\rho_{i}$ and a long tail. The jump-like increase of $P\left(\rho_{i}\right)$ for $\alpha \gtrsim 2$ signals the occurrence of a very narrow symmetric distribution centred around $\rho_{\mathrm{av}}$ and describes extended states. Finally Fig. 3 shows contours of the ratio $R(E)=\rho_{\text {ty }}(E) / \rho_{\text {av }}(E)$. Apparently all states become localised for $\alpha \lesssim 2$, whereas the width of the region of extended states saturates for $\alpha \gtrsim 5$.

In conclusion, we analysed the effect of impurity correlations on the localisation properties of $1 \mathrm{D}$ electron systems and showed that our model exhibits an insulator metal transition with increasing correlation strength.

Work was supported by NIC Jülich, HLRN BerlinHannover, and DFG under SPP 1073 (FE 398/1-3).

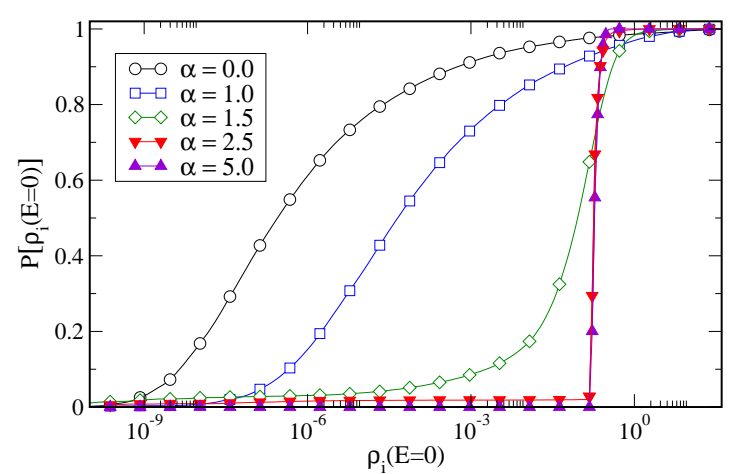

Fig. 2. Probability distribution $P\left(\rho_{i}\right)$ in the band-centre for different correlation strengths $\alpha$ (again $N=125000, M=32768$, $K_{r} \times K_{s}=32 \times 32$ ).

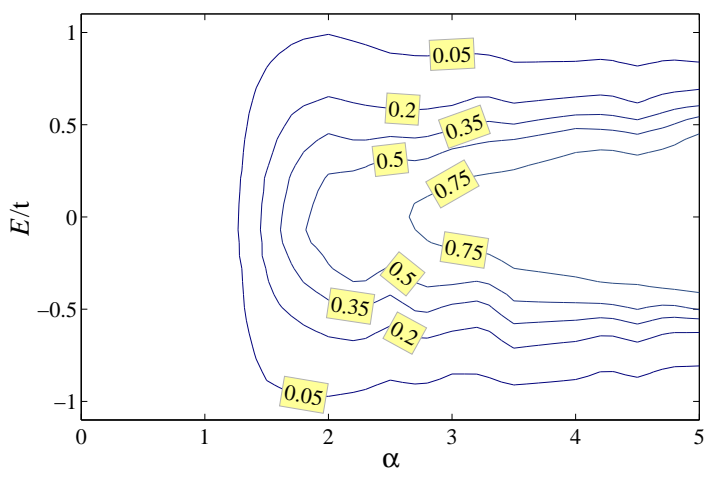

Fig. 3. Contour plot of $R$ in the correlation-energy plane for the Anderson model with correlated disorder on a linear chain with 125000 sites. In the calculation 32768 Chebyshev moments and $K_{r} \times K_{s}=128 \times 32$ realizations were used.

\section{References}

[1] P. Carpena et al., Nature 418, 955 (2002).

[2] V. L. Berezinskii, Zh. Eksp. \& Theor. Fiz. 65, 1251 (1973).

[3] J. C. Flores, J. Phys. Condens. Mat. 1, 8471 (1989); M. Hilke, Phys. Rev. Lett. 91, 226403 (2003).

[4] F. de Moura and M. L. Lyra, Phys. Rev. Lett. 81, 3735 (1998).

[5] M. Paczuski, S. Maslov, and P. Bak, Phys. Rev. E 53, 414 (1996).

[6] R. Abou-Chacra, P. W. Anderson, and D. J. Thouless, J. Phys. C 6, 1734 (1973); A. D. Mirlin and Y. V. Fyodorov, Phys. Rev. Lett. 72, 526 (1994).

[7] F. X. Bronold, A. Alvermann, and H. Fehske, Phil. Mag. 84, 673 (2004); A. Alverman et al., cond-mat/0406051

[8] G. Schubert, A. Weisse, H. Fehske, cond-mat/0309015/2. 